\title{
An Imported Case and an Infected Close Contact of the Omicron Variant of SARS-CoV-2 - Guangdong Province, China, December 13, 2021
}

\author{
Kuibiao Li ${ }^{1, x}$; Zhonghua Zheng, ${ }^{2, x}$; Xiang Zhao'; Qing Zeng'; Tengfei Zhou'; Qianfang Guo²; \\ Yao $\mathrm{Hu}^{2}$; Wenbo $\mathrm{Xu}^{3}$; Zhoubin Zhang ${ }^{1, * ;}$; Baisheng $\mathrm{Li}^{2, *}$
}

On November 27, 2021, a 67-year-old male returning from Canada via airplane was transferred to the central isolation hotel in Shanghai. During the quarantine, his 1st, 4th, 7th, and 14th days of specimens returned negative for severe acute respiratory syndrome coronavirus 2 (SARS-CoV-2), which causes coronavirus disease 2019 (COVID-19). On December 11, he completed 14 days of centralized isolation and returned to Guangzhou from Shanghai via airplane, then he was closed-loop transferred to Yuexiu District for a 7-day home isolation. On December 12, the local Community Health Service Center routinely collected his throat swab and the result returned to COVID-19 positive on December 13, 2021. He was transported to the Guangzhou Eighth People's Hospital for isolated treatment by negative pressure ambulance on the same day. The patient had two inflamed lungs according to the chest computed tomography (CT) examination, but his most recently updated condition was stable. An estimated 600 close contacts were identified and were placed in centralized isolation. On December 16, one of the close contacts, a 70-year-old female also tested positive for COVID-19. She lived on the lower floor of the same type of apartment as the imported case and spent 19 hours in the same building.

On December 13, 2021, the nasal swab was sequenced using both Nanopore GridION by Guangzhou CDC and Illumina MiniSeq by Guangdong CDC. The viral RNA was reverse transcribed and amplified using ULSEN ${ }^{\circledR} 2019-\mathrm{nCoV}$ Whole Genome Capture Kit (Beijing MicroFuture Technology Co., Ltd, Beijing, China, V-090418). Then, the sequencing libraries were prepared using the Illumina Nextera ${ }^{\circledR}$ XT Library Prep Kit (Illumina, Inc., San Diego, USA, FC-131-1001) and were conducted by Illumina sequencing. On December 14, 2021, the sequencing analysis concluded that the virus genome belonged to lineage B.1.1.529, Variant of Concern (VOC)/Omicron. The Omicron (B.1.1.529) variant was reportedly first discovered on November 9, 2021, in Botswana in southern Africa (1), and then gradually spread in South Africa, becoming the dominant strain in South Africa. On November 26, 2021, it was listed by the World Health Organization (WHO) as a VOC (2). The Omicron variant is a highly divergent variant with a high number of mutations, including position 26-32 in the spike protein, some of which are concerning and may be associated with immune escape potential and higher transmissibility. As of December 9, 2021, human infections with this variant had been identified in 63 countries (3).

Compared with the Wuhan reference sequence (EPI_ISL_402119) (4), the strain from the case displayed 59 nucleotide variation sites. The sequence reported on the evolutionary branch is most similar to those reported in England on December 4, 2021 (Figure 1). Furthermore, 48 amino acid mutation sites and 16 amino acid deletions were detected in the protein that corresponded to the features of the VOC/Omicron variant (B.1.1.529.1).

Researchers have established the existence of 2 variants of Omicron. The "standard" variant is now referred to as BA.1/B.1.1.529.1, and a second variant of Omicron known as BA.2/B.1.1.529.2. BA.2 has been nicknamed "Stealth Omicron" because it differs from the "standard" variety by not having the characteristic SGTF-causing deletion $(\Delta 69-70)(5)$. In this study, the variant has deletion $(\triangle 69-70)$ and belongs to sublineage BA.1/B.1.1.529.1.

Funding: The Basic Research Project of Key Laboratory of Guangzhou (No.202102100001), The Key Project of Medicine Discipline of Guangzhou (No.2021-2023-11).

doi: $10.46234 / \mathrm{ccdcw} 2021.265$

\# Corresponding authors: Zhoubin Zhang, gzcdczzb@gzcdc.org.cn; Baisheng Li, libsn@126.com.

\footnotetext{
Institute of Public Health, Guangzhou Medical University \& Guangzhou Center for Disease Control and Prevention, Guangzhou, Guangdong, China; ${ }^{2}$ Guangdong Provincial Center for Disease Control and Prevention, Guangzhou, Guangdong, China; ${ }^{3}$ National Institute for Viral Disease Control and Prevention, Chinese Center for Disease Control and Prevention, Beijing, China.

\& Joint first authors.
} 


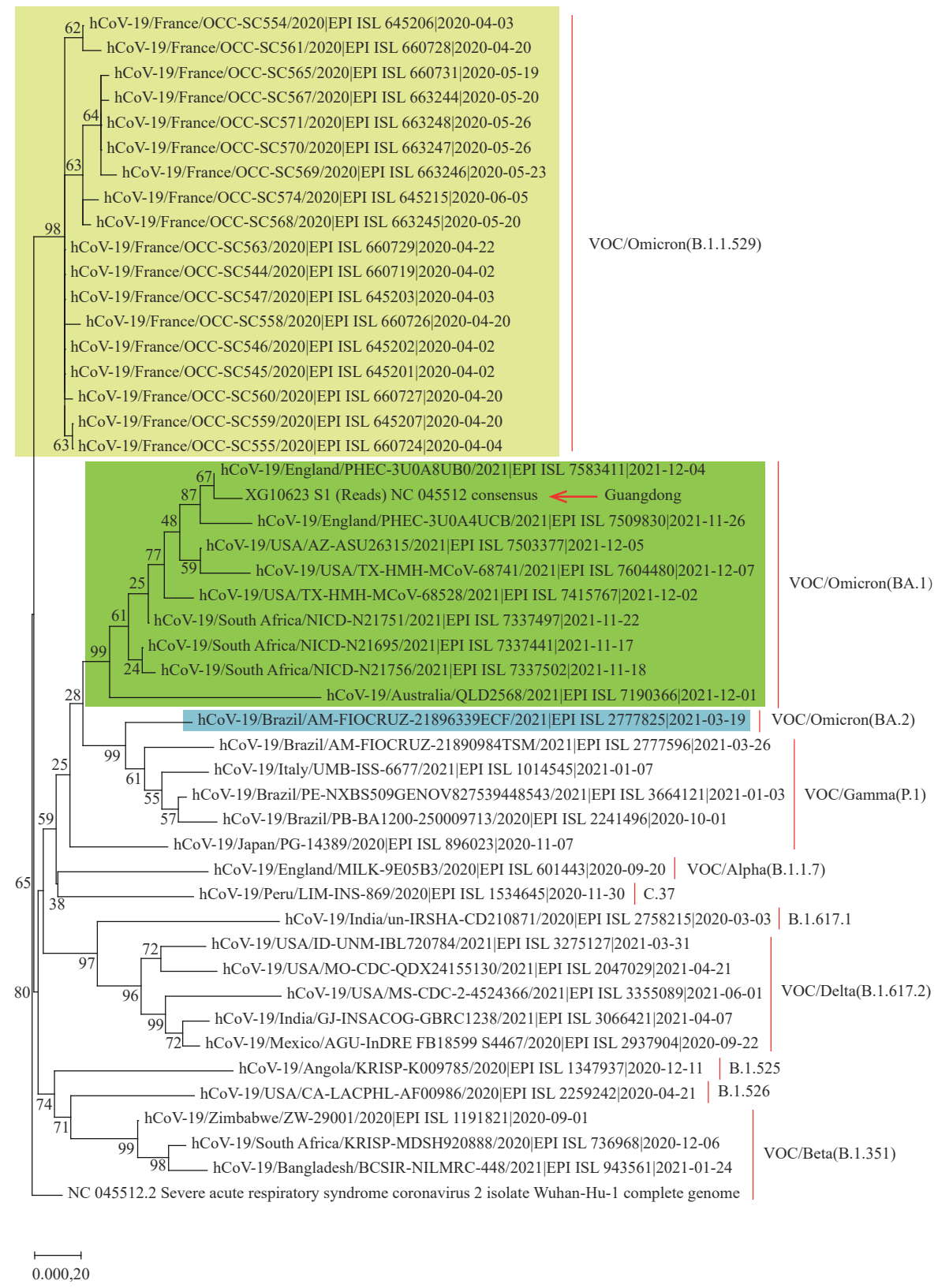

FIGURE 1. Phylogenetic tree based on the full-length genome sequences of the COVID-19 virus.

Note: The VOC/Omicron(B.1.1.529) variants are highlighted in yellow. The VOC/Omicron(BA.1) variants are highlighted in green and the Guangdong imported VOC/Omicron(BA.1) variant is marked with an arrow. The VOC/Omicron(BA.2) variant is highlighted in blue. The 11 distinguished COVID-19 mutants are marked on the right. Abbreviations: VOC=variant of concern; COVID-19=coronavirus disease 2019.

Submitted: December 16, 2021; Accepted: December 17, 2021

\section{REFERENCES}

1. Callaway E. Heavily mutated Omicron variant puts scientists on alert. Nature. 2021;600(7887):21. http://dx.doi.org/10.1038/d41586-02103552-w.

2. World Health Organization. Classification of Omicron (B.1.1.529): SARS-CoV-2 Variant of Concern. https://www.who.int/news/item/2611-2021-classification-of-omicron-(b.1.1.529)-sars-cov-2-variant-of- concern. [2021-12-14].

3. World Health Organization. COVID-19 Weekly Epidemiological Update-7 December 2021. https://www.who.int/publications/m/item/ weekly-epidemiological-update-on-covid-19---7-december-2021. [202112-14].

4. Wu F, Zhao S, Yu B, Chen YM, Wang W, Song ZG, et al. A new coronavirus associated with human respiratory disease in China. Nature 2020;579(7798):265. http://dx.doi.org/10.1038/s41586-020-2008-3.

5. Guardian. Scientists find 'stealth' version of Omicron that may be harder to track. https:/www.theguardian.com/world/2021/dec/07/scientistsfind-stealth-version-of-omicron-not-identifiable-with-pcr-test-covidvariant. [2021-12-14]. 